\title{
Effect of Interaction Design on the Human Experience with Interactive Reinforcement Learning
}

\author{
Samantha Krening \\ Georgia Institute of Technology \\ skrening@gatech.edu
}

\author{
Karen M. Feigh \\ Georgia Institute of Technology \\ karen.feigh@gatech.edu
}

\begin{abstract}
A goal of interactive machine learning (IML) is to enable people with no machine learning knowledge to intuitively teach intelligent agents how to perform tasks. This study investigates how three factors of the design of an interactive reinforcement learning agent - generalization through time, immediacy, and a time delay - impact the user's experience with the agent. We conducted a human-subject experiment in which people trained four agents with different interaction designs to play a simple game using verbal instruction. All agent variations were modified versions of the Newtonian Action Advice algorithm, an interactive reinforcement learning agent that learns from verbal advice like, "go left." The results show that both a time delay and probabilistic interface created poor user experiences. This is particularly important for IML designers, because the current algorithms almost universally are probabilistic and do not immediately respond to the human's input.
\end{abstract}

\section{Author Keywords}

human-agent interaction; interaction design; interactive machine learning; reinforcement learning; human factors; natural language interface

\section{CCS Concepts}

-Human-centered computing $\rightarrow$ Human computer interaction (HCI); Natural language interfaces; Interaction design; -Computing methodologies $\rightarrow$ Reinforcement learning;

\section{INTRODUCTION}

The field of interactive machine learning (IML) works toward making it possible for everyday people who are not experts in machine learning to naturally and intuitively teach intelligent agents to perform tasks. If A.I. is to be used universally in everyday life, intelligent agents must be designed to interact with and learn from non-expert end-users using methods that are user-friendly for people. It is not practical to train people to use intelligent agents; rather, we design intelligent agents that can easily be trained by people.

\footnotetext{
Permission to make digital or hard copies of all or part of this work for personal or classroom use is granted without fee provided that copies are not made or distributed for profit or commercial advantage and that copies bear this notice and the full citation on the first page. Copyrights for components of this work owned by others than the author(s) must be honored. Abstracting with credit is permitted. To copy otherwise, or republish, to post on servers or to redistribute to lists, requires prior specific permission and/or a fee. Request permissions from permissions@ acm.org.

DIS '19, June 23-28, 2019, San Diego, CA, USA

(C) 2019 Copyright held by the owner/author(s). Publication rights licensed to ACM. ISBN 978-1-4503-5850-7/19/06. . 15.00

DOI: https : //doi .org/10.1145/3322276. 3322379
}

This study focuses on the user experience of an Interactive Reinforcement Learning agent. Interactive RL algorithmic design generally ignores the human experience. Some IML researchers have noticed how human responses are not the same as oracles [1], yet moving beyond oracles to verification with human subjects has not been made common practice with interactive RL agents. Algorithms are verified with oracles, i.e. simulated human input, rather than human-in-the-loop studies, and performance is compared with objective machine learning metrics such as reward and training time [24, 30, 6, 3]. While oracle testing is necessary, it does not provide a way to measure how humans react to the agent. For example, an oracle can never get frustrated with an agent. Creating a positive human experience is important because individuals who experience frustration while interacting with a robot are unlikely to interact with the robot in the future [23].

This work builds on our previous study that analyzed human satisfaction with IML agents compared the human experience of two verbal interaction methods: advice (e.g. "right, left") and critique (e.g. "good, bad") $[15,14]$. The study found that the Newtonian Action Advice (NAA) algorithm created a better user experience than a critique Policy Shaping agent. From text responses provided by participants, the study identified several characteristics of the interaction design of IML algorithms they suspected would impact the user experience, including immediacy and a deterministic interaction. The critique and action advice agents differed in many ways including whether they allowed instructions for future actions, how immediately action selection is effected by human input, the nature of the input, and how the input was used. While the original study determined that interaction algorithms impact the human's experience, the study did not allow a systematic analysis of to what extent specific attributes of the interaction algorithm impact the human's experience. This paper delves deeper into specific design decisions to understand the impact of each decision on the human's satisfaction with the agent.

This paper focuses on the question: Do the following three factors of an agent's interaction design affect the person's experience with the agent: 1) generalization through time, 2) time delay, and 3) a probabilistic interface? If so, to what extent? If we can isolate specific design decisions that impact the user's experience, IML researchers can design algorithms that create a positive user experience. We hypothesized the human's experience to be improved by designing an interaction algorithm to: have a minimal delay between when human instructions are given and used by the agent; incorporate the human input in a non-probabilistic manner; and generalize the 
human's input through time so the human does not need to provide as much instruction.

We performed a repeated measures human-subjects experiment in which participants taught four agents how to play a game with different interaction designs. All four agent variations were based on the Newtonian Action Advice algorithm (NAA), which is an IML algorithm that enables people to teach an agent using verbal action advice (i.e. verbal demonstration) such as "move down," and "go left."

\section{BACKGROUND}

\section{Reinforcement Learning}

Reinforcement learning (RL) is a branch of machine learning in which intelligent agents learn from the environment which actions to take by receiving a signal of rewards and punishments [31]. RL has been a topic of study in behavioral psychology [31, 27]. B. Skinner compared the evolution of living things through natural selection with the shaping of individual behavior through reinforcement [28]. People will likely repeat an action in a circumstance if they receive positive reinforcement, and will likely not repeat the action if given negative reinforcement. People learn by interacting with their environment, so we chose a machine learning algorithm that replicates this process.

Most RL algorithms are modeled as Markov Decision Processes (MDPs), which learn policies by mapping states to actions such that the agent's expected reward is maximized. An MDP is a tuple $(S, A, T, R, \gamma)$ that describes $S$, the states of the domain; $A$, the actions the agent can take; $T$, the transition dynamics describing the probability that a new state will be reached given the current state and action; $R$, the reward earned by the agent; and $\gamma$, a discount factor in which $0 \leq \gamma \leq 1$.

Bayesian Q-Learning was the (MDP-based) underlying RL agent used for all four variations of the Newtonian Action Advice agent in this study. Bayesian Q-Learning is an RL algorithm in which the utility of state-action pairs are represented as probabilistic point estimates of the expected long term discounted reward [5].

\section{Learning from Critique}

Many IML algorithms learn from critique. Initially, critique was incorporated into RL as a reward signal [9]. This was shown to be less than optimal because people provide an unpredictable signal and stop providing critique once the agent learns the task [10]. Later, critique was used as policy information rather than critique $[12,33]$. An example of this is Policy Shaping, an IML algorithm that enables a human to teach an agent using critique, which is incorporated into a Bayesian Q-learning agent as policy information [6]. A recent study showed that Policy Shaping using critique creates a worse user experience than an advice algorithm such as Newtonian Action Advice [15].

\section{Learning from Action Advice}

For the agent that learns from advice in this experiment, we used Newtonian Action Advice (NAA) [16]. NAA is based on Newton's first law: objects in motion stay in motion unless acted on by an external force. The NAA model treats action advice provided by the human as an external force acting on the agent.

When action advice (ex: "Move left") is provided by the person, the agent will immediately move in the direction of the advice, supplanting the RL agent's action selection method. The model contains friction that opposes the advice and 'slows down' the agent's need to follow the human's advice. The friction ensures that the agent will resume the underlying RL algorithm's action selection policy after some amount of time. For example, if the teacher provides advice to move down and the model's friction parameter is 5 steps, the agent will deterministically follow the advice and move down for 5 time steps. After 5 time steps have elapsed, the agent will return to its action selection method. NAA uses Bayesian Q-learning as the underlying RL algorithm.

There are many other forms of advice. Some advice connects a condition to an action [20], another connects conditions to rewards [19], while another learns policies using demonstrations and advice [2]. Many link conditions to researcher-defined higher-level actions $[20,18,11]$. People provide a relative preference of actions in [20], whereas [17] learns the order of preferred actions. [26] learns multiple interpretations of instructions. [32] represents natural language commands as probabilistic graphical models, and [21] parses language into a graphical representation and then to primitive actions. Most forms of advice are permanently influenced by the advice [18, 20]; NAA differs because the advice can be overwritten by new advice in the future.

\section{Automatic Speech Recognition (ASR)}

All four agents used in this study learn from verbal action advice - the human teacher speaks to the computer giving instructions such as "left." ASR is how the human's verbal instructions are transcribed to written text. This experiment used the Sphinx ASR software [8].

\section{METHOD}

We conducted a repeated-measures human-subject experiment in which we investigated the effect of four interaction algorithm variations on the human's satisfaction with the agent. The study collected data from 24 participants who were not experts in machine learning. The age of the participants varied from 18-65 years old.

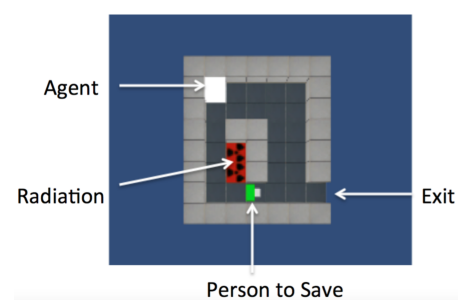

Figure 1: Radiation World Initial Condition

The task required participants to teach each agent to rescue a person in Radiation World, which is a game developed in the unity minecraft environment as shown in Figure 1 and has been 
used in other IML studies $[14,16,15]$. In the experimental scenario, there has been a radiation leak and an injured person is located somewhere in the grid unable to move. The agent must find and rescue the person, take him to the exit, all while avoiding the radiation. Light gray cells represent walls, while dark gray cells are areas where the agent can safely move. The task is complete if the agent takes the person to the exit. The task is failed if the agent enters the radiation or exits without the person. The task is repeated for several training episodes; participants were told to stop training when either the agent was performing as they intended or the participant was too frustrated to continue or wanted to stop for any reason. Consequently, the training time varied for each participant and interaction algorithm. After a participant finished training an agent, the participant completed a questionnaire concerning the experience. At the end of the experiment, participants were asked if they had any questions or additional thoughts concerning the experiment in an exit interview.

All four agents learned from verbal instruction that can be thought of as verbal demonstrations. The verbal instructions were transcribed to text using ASR software. After language processing, the processed instructions were sent to one of the NAA interaction algorithm variations.

People were instructed to tell the agent to move in a desired direction. For example, if participants wanted the agent to move down, they should say, "down." The only four words recognized by the action advice agents were, "up," "down," "left," and "right." The action advice agent used the Newtonian Action Advice algorithm to incorporate the advice with the Bayesian Q-learning algorithm's action selection.

\section{Newtonian Action Advice Algorithm and Variations}

During this study, each participant trained four agents in a different order. Each agent was a variation of the NAA algorithm.

\section{Algorithm 1: Newtonian Action Advice, 5 steps}

The first algorithm developed for this study was the same NAA algorithm used in [15]. The "5 steps" refers to the amount of time steps a human's advice would be followed before the NAA agent reverted back to its underlying action selection method. If a participant told the agent to move left, the agent would move left for five time steps unless the participant interrupted to provide new, superseding advice.

\section{Algorithm 2: Newtonian Action Advice, 1 step (no generaliza-}

\section{tion)}

The second algorithm developed for this study differed from the first by following advice for one time step instead of five. This results in participants being required to provide advice more often. For example, if the participant wanted the agent to move down five steps and then right four steps, the participant would need to say, "Down, down, down, down, down, right, right, right, right." This algorithm variation is testing whether no generalization of advice through time impacts the user's experience. One reason for testing this variation is that many algorithms that learn from demonstrations treat each demonstration as a strict (state, action) pair and do not generalize either through time or similar states.
Algorithm 3: Newtonian Action Advice, 5 steps, probabilistic The third algorithm developed for this study differed from the first by incorporating advice in a probabilistic manner. Each time new advice was given by the person, the agent would follow the advice with a $60 \%$ probability or choose an action based on the agent's action selection method with a $40 \%$ probability.

A certain level of unpredictability may be necessary for a fun user experience [4]. However, unpredictability can induce stress in humans [22]. Our previous study found that a Policy Shaping agent that integrates the human's critique with the RL agent in a probabilistic manner resulted in a worse user experience than an NAA agent that incorporates the human's advice in a deterministic manner $[15,14]$. This Probabilistic algorithm variation is testing whether, and to what extent, using advice in a probabilistic manner impacts the user experience.

A Human Factors expert may think it is obvious that a probabilistic interface is a poor idea, while a Machine Learning engineer might never consider another option. Modern ML algorithms universally rely on probability and statistical modeling - it is how we account for uncertainty and noise in real-world systems. When ML researchers hear the word 'deterministic', they will be reminded of the model-based paradigm of the 1970s; this approach only worked in very limited domains with highly accurate models, no uncertainty, and deterministic systems. The field of ML has come a long way since then, and ML researchers will be hesitant to use anything reminiscent of old methods.

Probability is used a great deal in RL, not just for statistical modeling but determining what actions to choose. In Reinforcement Learning, the agent needs to balance between exploring new solutions that might earn a higher reward and exploiting the policy the agent thinks is best based on experience. $\varepsilon$-greedy is one method of balancing between exploration and exploitation. The agent has some value of $\varepsilon(0,1)$. At each time step, the agent choose a random value $(0,1)$. If the value is greater than $\varepsilon$, the agent exploits the learned policy; otherwise, the agent explores by choosing a random action. The value of $\varepsilon$ may be instantiated quite high (ex: 0.95), but then smoothly decayed through time so the agent explores a great deal in the beginning but then settles to a learned policy after a while. This type of decay curve is fairly common for other parts of RL algorithms, including the learning rate. When incorporating human input, RL researchers may want to do something similar, largely because they are so familiar with this type of logic. They may build a system that uses human input with a greater probability early on in the learning process, like human-directed exploration. In the case of Policy Shaping, human input is represented as a table of probabilistic point estimates, just like the underlying utility estimate; both human and agent probabilities are multiplied together to choose the 'best' action. These design decisions are made to allow the human critique to be incorporated seamlessly and mathematically into the RL algorithm; however, no thought is given to how these decisions impact the interaction with and experience of the human. 
RL researchers may not shy away from using human input in a probabilistic manner unless studies like this paper show it adversely impacts the user experience. We want to demonstrate that we can create a deterministic interaction between the human and agent to improve the human experience while keeping the statistical nature of the underlying RL agent.

Algorithm 4: Newtonian Action Advice, 5 steps, time delay The fourth algorithm developed for this study differs from the first by introducing a two second time delay between when advice was provided by the participant and when the agent followed the advice. Users can adapt to frequent, expected time delays [13], and users tend to prefer delays of less than one second [25]. However, time delays can improve performance on difficult tasks [29]. This algorithm variation is testing whether, and to what extent, the immediacy of using advice impacts the user experience.

When designing an advice algorithm, a researcher may not purposefully build in an extended time delay. However, the researcher may not make an effort to minimize a time delay unless studies like this one show it greatly impacts the user experience. Many IML algorithms, such as those that learn from critique, do not have a method of immediately using the human's instruction.

\section{Experimental Procedure}

The procedure followed by all participants is provided below. All participants experienced the algorithm variations in a different order.

1. Greeting and Introduction

2. For each of the 4 agents: Instructions for training the agent. Train the agent. Questionnaire about experience of training the agent.

3. Free response questions about the experience of training the agents.

\section{Human Experience Measures}

The Human Experience measures were created as a modified version of the NASA-TLX questionnaire [7]. The answers to all questions regarding the human experience were collected using a sliding bar in which the selected value to the tenths decimal place was shown to the participant.

Paired tests. Immediately after training an agent, the participants were asked to score the intelligence of the agent on a continuous scale from [0:10]. A value of 0 indicated that the agent was not intelligent, while 10 meant very intelligent. The same scale of [0:10] was used for four additional metrics: performance, frustration, transparency, and immediacy. Values of 0 corresponded to poor performance, low frustration, non-transparent use of feedback, and a slower response time. Values of 10 meant excellent performance, high frustration, clear use of feedback, and an immediate response time, respectively.

Explanation of Responses. After training both agents, in item 3 of the procedure participants were given the option to explain what factors impacted their experience of working with the agents. For example, participants were asked, "If one agent was more frustrating than another, what made you feel that way?" These written responses were entirely free form with no priming or options provided by the experimenter. The purpose of these explanations is to explore why the interaction algorithm impacts the human's experience. The resultant set of design characteristics that affect the person's relationship with the ML agent allows us to analyze each of these characteristics thoroughly in future work and eventually use the characteristics to direct the design of IML algorithms.

\section{Objective ML measures}

While participants were training the agents, objective performance metrics were logged to data files. The training time and reward earned per episode were measured as continuous data. The amount of human input provided and the number of actions it took to complete an episode were measured as ordinal count data.

\section{RESULTS AND DISCUSSION}

The algorithm variations differed across the board in terms of both human factors and objective metrics. The NAA 5 steps and NAA 1 step algorithms performed the best, followed by the Probabilistic variation. Overall, the Time Delay variation performed the worst.

\section{Human Factors Metrics}

Figure 2 shows the boxplots of all the human factors results. For all human factors metrics except frustration, higher values represent a better human experience (better performance, greater transparency, more immediate, and more intelligent). For frustration, higher values indicate a higher level of frustration, and therefore a worse human experience. The 5 steps and 1 step agents performed better than the Probabilistic and Time Delay variations on every human factors metric.

Figure 3 shows the distribution of responses for the human factors metrics. A line is drawn at the middling value of 5 for each of the human factors. Both the 5 steps and 1 step (no generalization) variations show distributions that are skewed toward a positive human experience: high transparency, perceived performance, intelligence, immediacy, and low frustration. The distributions for the Probabilistic variation are shifted toward a middling and poor user experience. The Time Delay distributions are skewed toward a negative user experience and are almost the opposite of the 5 steps and 1 step variations; the distributions show that participants thought the Time Delay variation was not transparent, not intelligent, responded slowly to advice, had middling performance, and was highly frustrating.

Each of the violin plots (Figures 4 - 6) show the data distribution drawn around a boxplot.

\section{Frustration}

Figure 4 shows the 5 steps and 1 step agents resulted in the lowest frustration. The Time Delay variation was the most frustrating, followed closely by the Probabilistic agent. 


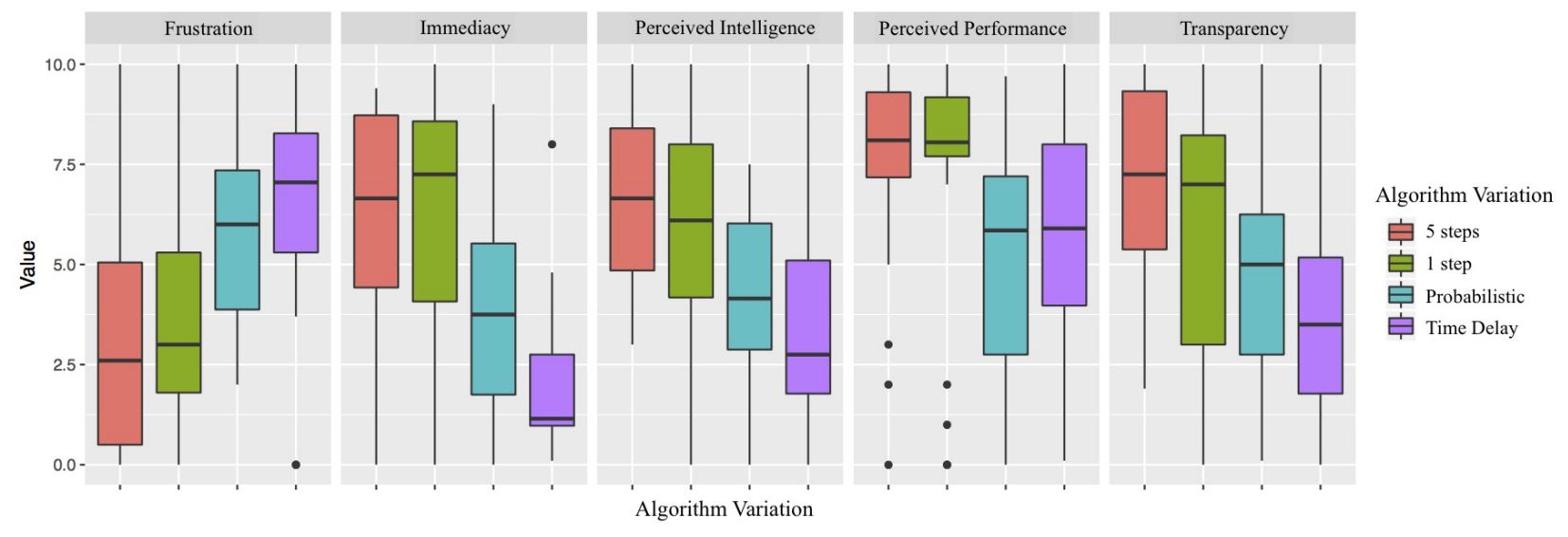

Figure 2: Box Plots Showing Human Factors Results For NAA Variations

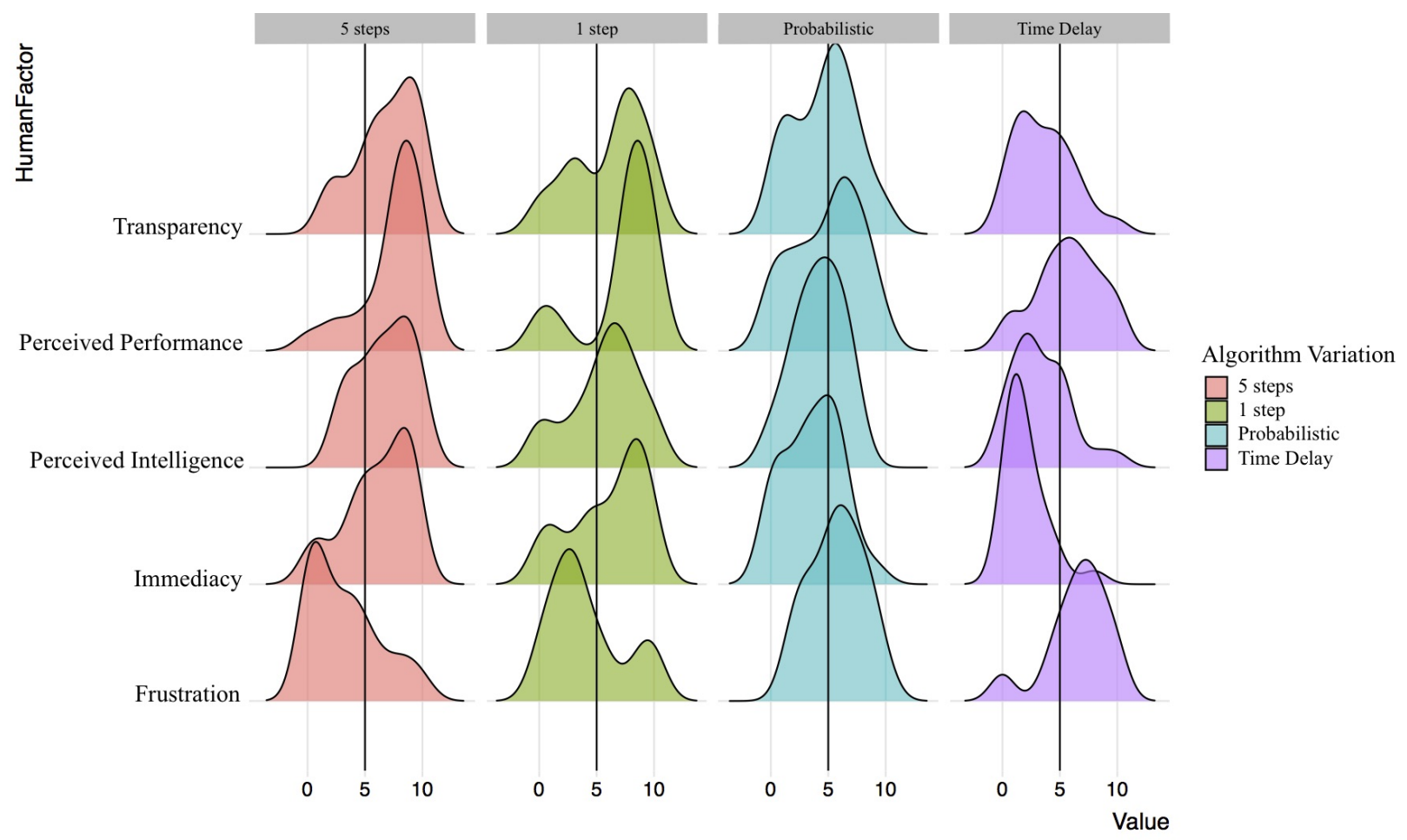

Figure 3: Distributions of Human Factors Results For NAA Variations 
Table 1: Frustration: Repeated-Measures ANOVA

\begin{tabular}{llllll}
\hline & SS & $S S E$ & $d f$ & $F$ & $\operatorname{Pr}(>F)$ \\
\hline Intercept & 2325.59 & 249.78 & 23 & 214.1453 & $3.836 \mathrm{e}-13$ \\
Algorithms & 167.35 & 490.22 & 69 & 7.8518 & 0.0001397 \\
\hline
\end{tabular}

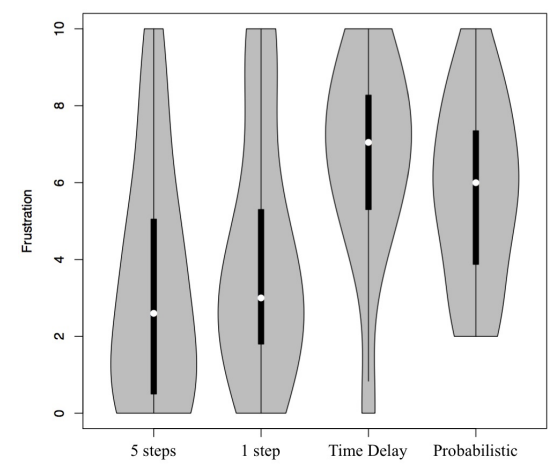

Figure 4: Frustration Results for NAA Variations

A one-way repeated measures ANOVA shows that frustration is not equal across all NAA algorithm variations (Table 1). A post-hoc analysis of a series of paired t-tests shows the 5 steps and 1 step variations are equal in frustration. The Probability and Time Delay variations are equal in frustration. However, the 5 steps and 1 step variations are less frustrating than the Probability and Time Delay variations.

The profile of the 5 steps and 1 step variations being equal to each other and better than the Probability and Time Delay variations will be repeated on all of the remaining human factors except Immediacy.

\section{Perceived Performance}

Table 2: Perceived Performance: Repeated-Measure ANOVA

\begin{tabular}{llllll}
\hline & SS & $S S E$ & $d f$ & $F$ & $\operatorname{Pr}(>F)$ \\
\hline Intercept & 3902.8 & 290.21 & 23 & 309.3039 & $7.814 \mathrm{e}-15$ \\
Algorithms & 113.0 & 490.85 & 69 & 5.2971 & 0.002405 \\
\hline
\end{tabular}

Figure 5a shows the 5 steps and 1 step agents were clustered around a very high perceived performance. The Time Delay and Probabilistic variations had more variation and were overall seen as performing worse.

A one-way repeated measures ANOVA shows that perceived performance is not equal across all NAA variations (Table 2). A post-hoc analysis of a series of paired t-tests shows the 5 steps and 1 step variations are perceived to have equal performance. The Probability and Time Delay variations are equal in perceived performance. However, the 5 steps and 1 step variations are perceived to perform better than the Probability and Time Delay variations.
Table 3: Transparency: Repeated-Measures ANOVA

\begin{tabular}{llllll}
\hline & SS & $S S E$ & $d f$ & $F$ & $\operatorname{Pr}(>F)$ \\
\hline Intercept & 2715.8 & 293.78 & 23 & 212.619 & $4.133 \mathrm{e}-13$ \\
Algorithms & 151.1 & 423.63 & 69 & 8.204 & $9.584 \mathrm{e}-05$ \\
\hline
\end{tabular}

Transparency

Figure $5 \mathrm{~b}$ shows the 5 steps agent was seen to be the most transparent, with no participants scoring less than 2 . The 1 step agent also scored as very transparent. The Probabilistic variation was seen as middling in terms of transparency. The Time Delay variation was seen as the least transparent, with an average less than 4 .

A one-way repeated measures ANOVA shows that transparency is not equal across all NAA variations (Table 3). A post-hoc analysis of a series of paired t-tests shows the 5 steps and 1 step variations are perceived to be equally transparent. The Probability and Time Delay variations are equal in transparency. However, the 5 steps and 1 step variations are perceived to be more transparent than the Probability and Time Delay variations.

\section{Perceived Intelligence}

Table 4: Perceived Intelligence: Repeated-Measures ANOVA

\begin{tabular}{llllll}
\hline & SS & $S S E$ & $d f$ & $F$ & $\operatorname{Pr}(>F)$ \\
\hline Intercept & 2379.05 & 190.0 & 23 & 287.987 & $1.679 \mathrm{e}-14$ \\
Algorithms & 161.12 & 398.3 & 69 & 9.304 & $3.023 \mathrm{e}-05$ \\
\hline
\end{tabular}

Figure $5 \mathrm{c}$ shows the 5 steps agent had the highest mean score of perceived intelligence, with no participants responding less than a score of 3 , followed closely by the 1 step agent. The Probabilist variation had a higher mean than the Time Delay agent, but the Probabilistic agent had no scores greater than 7.5 .

A one-way repeated measures ANOVA shows that perceived intelligence is not equal across all NAA variations (Table 4). A post-hoc analysis shows the 5 steps and 1 step variations are equal in perceived intelligence. The Probability and Time Delay variations are equal in perceived intelligence. However, the 5 steps and 1 step variations are perceived to be more intelligent than the Probability and Time Delay variations. Immediacy

Table 5: Immediacy: Repeated-Measures ANOVA

\begin{tabular}{llllll}
\hline & SS & $S S E$ & $d f$ & $F$ & $\operatorname{Pr}(>F)$ \\
\hline Intercept & 1893.04 & 159.93 & 23 & 272.236 & $3.058 \mathrm{e}-14$ \\
Algorithms & 284.45 & 486.21 & 69 & 13.456 & $5.230 \mathrm{e}-07$ \\
\hline
\end{tabular}

Figure 6 shows the 5 steps and 1 step agents were seen as implementing a human's advice very quickly. The Probabilistic 


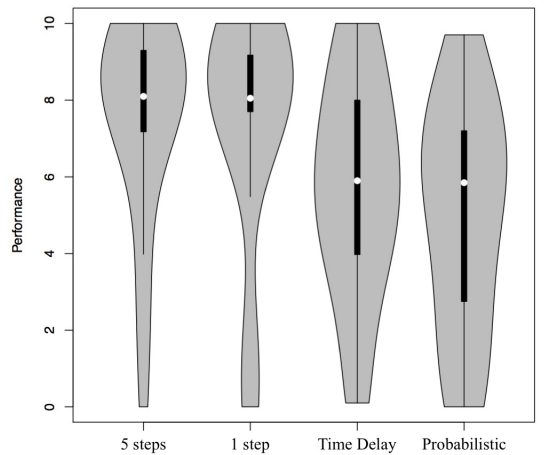

a: Perceived Performance Results for NAA Variations

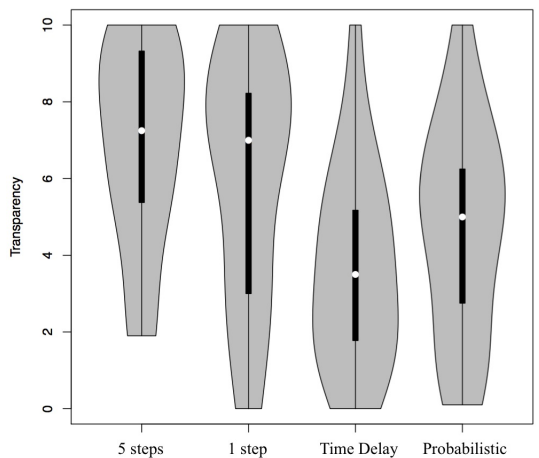

b: Transparency Results for NAA Variations

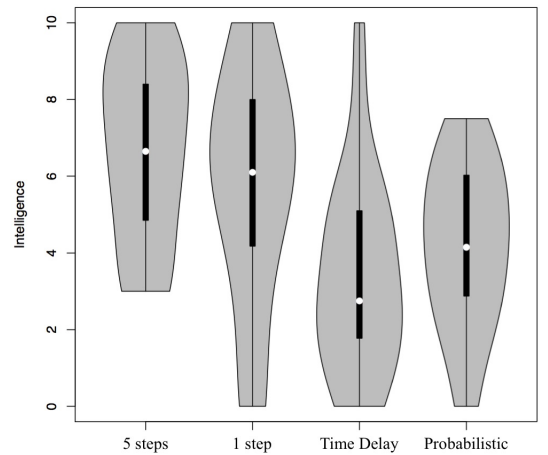

c: Perceived Intelligence Results for NAA Variations

Figure 5: Violin plots for Perceived Performance, Transparency, and Perceived Intelligence.

variation had middling performance. The Time Delay variation was almost universally seen as responding to advice very slowly, with a mean less than 2 .

A one-way repeated measures ANOVA shows that immediacy is not equal across all NAA variations (Table 5). The 5 steps and 1 step variations are perceived to respond to advice equally quickly. However, the Time Delay variation is not equal to the Probability variation, which is in turn not equal to the 5 steps algorithm. People found the Time Delay variation to respond the slowest to input, followed by the Probability variation. The 5 steps and 1 step algorithms responded the fastest.

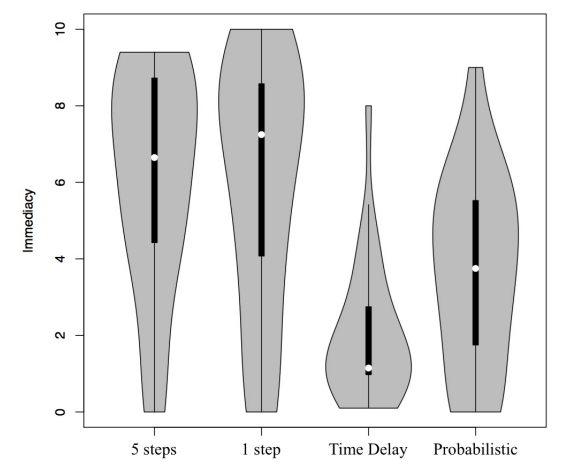

Figure 6: Immediacy Results for NAA Variations

It is both relieving and slightly unexpected that participants found the Time Delay variation to respond the slowest to input by a significant amount. Most participants did not realize the nature of the Time Delay variation; these results show that they were aware this variation did not respond immediately to input, but most could not figure out that a repeated time delay was the factor being tested in the Time Delay variation.

\section{Objective Metrics}

The 5 steps and 1 step agents performed approximately equally in terms of objective metrics; they earned a higher reward in less time than the Time Delay and Probabilistic variations. People provided less advice for the 5 steps algorithm than the others.

\section{Avg Training Time}

Table 6: Training Time: Repeated-Measures ANOVA

\begin{tabular}{llllll}
\hline & SS & $S S E$ & $d f$ & $F$ & $\operatorname{Pr}(>F)$ \\
\hline Intercept & 96617 & 2595.9 & 20 & 744.383 & $<2.2 \mathrm{e}-16$ \\
Algorithms & 2993 & 4163.8 & 60 & 14.375 & $3.633 \mathrm{e}-07$ \\
\hline
\end{tabular}

In Figure 7, the 1 step agent has the lowest mean training time, followed closely by the 5 steps agent. The Time Delay algorithm took the longest for people to train.

A one-way repeated measures ANOVA shows that training time is not equal across all NAA variations (Table 6). A posthoc analysis of a series of paired t-tests shows the 5 steps and 1 step variations are equal in training time. The Probability variation has a longer training time than the 5 steps and 1 step variations. The Time Delay variation has the longest training time.

Avg Amount of Advice Given by Human

Table 7: Amount of Advice: Repeated-Measures ANOVA

\begin{tabular}{llllll}
\hline & SS & SSE & $d f$ & $F$ & $\operatorname{Pr}(>F)$ \\
\hline Intercept & 7796.3 & 457.17 & 20 & 341.068 & $4.916 \mathrm{e}-14$ \\
Algorithms & 340.2 & 687.12 & 60 & 9.903 & $2.135 \mathrm{e}-05$ \\
\hline
\end{tabular}

In Figure 7, the 5 steps agent required the least amount of advice from the human during training. Compared to the 5 steps algorithm, people gave more advice to the 1 step agent; this is expected because the lack of generalization through time in the 1 step agent means the person needs to repeat themselves. The Probabilistic variation was given more advice than the 1 step agent. The Time Delay variation was given the most advice out of all the variations; this is interesting because the Time Delay agent can theoretically be trained using the same amount of advice as the 5 steps algorithm, but people either did not realize a time delay existed or refused to wait for their advice to be enacted and kept providing advice. 


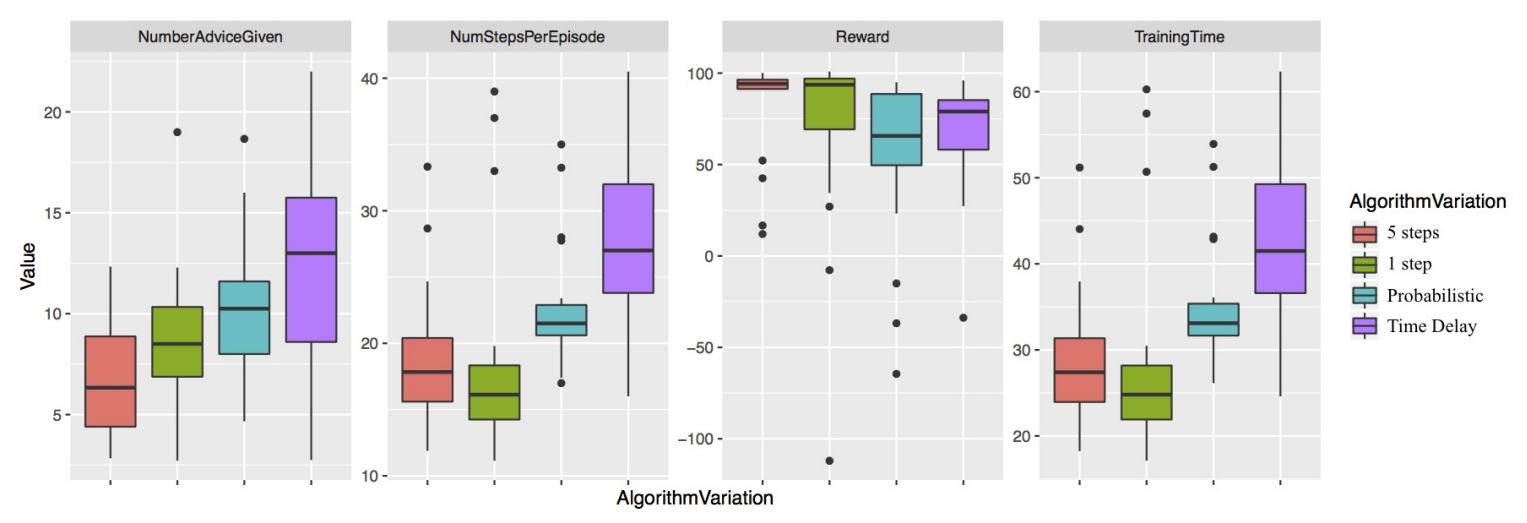

Figure 7: Objective Metric Results for average values of NAA Variations

A one-way repeated measures ANOVA shows that amount of advice given by the human is not equal across all NAA variations (Table 7). In fact, a post-hoc analysis shows none of the variations resulted in an equal amount of advice. People provided the least amount of input while training the 5 steps variation, followed by the 1 step, Probability, and Time Delay variations.

\section{Avg Reward}

Table 8: Earned Avg Reward: Repeated-Measures ANOVA

\begin{tabular}{llllll}
\hline & SS & $S S E$ & $d f$ & $F$ & $\operatorname{Pr}(>F)$ \\
\hline Intercept & 391159 & 43853 & 20 & 178.3938 & $2.004 \mathrm{e}-11$ \\
Algorithms & 8152 & 80719 & 60 & 2.0198 & 0.1207 \\
\hline
\end{tabular}

In Figure 7, the 5 steps agent earned the highest reward with the least variation, followed by the 1 step (no generalization) algorithm. The Time Delay had the third highest average reward per episode. The Probabilistic variation earned the lowest average reward.

A one-way repeated measures ANOVA shows that average reward per episode is statistically equal across all NAA variations (Table 8). Most algorithms are compared using the reward earned by the agent. The human factors results show that the user experience is very different for the variations, even if the average reward is statistically equal.

\section{Avg Number of Steps Agent took to Complete Each Episode}

Table 9: Number of Steps: Repeated-Measures ANOVA

\begin{tabular}{llllll}
\hline & SS & $S S E$ & $d f$ & $F$ & $\operatorname{Pr}(>F)$ \\
\hline Intercept & 40815 & 1084.8 & 20 & 752.478 & $<2.2 \mathrm{e}-16$ \\
Algorithms & 1268 & 1747.0 & 60 & 14.514 & $3.225 \mathrm{e}-07$ \\
\hline
\end{tabular}

In Figure 7, the 1 step agent has the lowest mean number of steps to complete each episode, followed closely by the 5 steps agent. The Time Delay algorithm took the greatest number of steps to complete each episode with the greatest variation.
This data distribution is very similar to training time since each game step takes approximately the same amount of time to execute.

A one-way repeated measures ANOVA shows that average amount of steps the agent took to complete each level is not equal across all NAA variations (Table 9). A post-hoc analysis shows the 5 steps and 1 step variations are equal in steps to completion. The Probability variation has a larger average steps to completion than the 5 steps a 1 step variations. The Time Delay variation has the largest average steps to completion.

\section{Long Responses from Participants}

At the very end of the experiment, participants were given a chance to provide text responses about their experiences interacting with the agents. According to the responses, several aspects of the agent's interaction impacted the participants' perceived intelligence and transparency as well as frustration with the agent. There were many commonalities across the participants' responses; we have organized and tallied these responses in Table 10.

The four aspects of the agent's interaction that were most often cited by participants as impacting frustration, intelligence, and transparency were: 1) compliance with input (whether the agent followed the person's advice), 2) immediacy (whether the agent immediately followed the person's advice), 3) amount of instruction the person had to provide, and 4) randomness (whether the agent was perceived to act in a random or deterministic manner).

The question prompts for the long responses are listed below. The participant quotes given in the following sections are answers to these question prompts.

- If one agent was more frustrating than another, what made you feel that way?

- If one agent was more intelligent than another, what made you think that?

- If one agent used your feedback in a clearer way than another, what made you think that? 
Table 10: Percentage of participants who mentioned in their long responses certain features that contributed to the user experience.

\begin{tabular}{c|c|c|c}
\hline \multirow{2}{*}{ Feature } & \multicolumn{3}{|c}{ \% Mentioned Feature Impacted HF } \\
\cline { 2 - 4 } & Frustration & Intelligence & Transparency \\
\hline Compliance & 71 & 54 & 23 \\
\hline Immediacy & 38 & 54 & 50 \\
\hline Less Instruction & & 29 & 27 \\
\hline Randomness & 21 & & 23 \\
\hline $\begin{array}{c}\text { Improvement } \\
\text { through time }\end{array}$ & & 17 & \\
\hline Memory & & 9 & 14 \\
\hline $\begin{array}{c}\text { Had to } \\
\text { repeat myself }\end{array}$ & 13 & & \\
\hline Effort & 9 & & \\
\hline Frustration & & 4 & \\
\hline Transparency & 4 & & \\
\hline
\end{tabular}

\section{Compliance with input}

Compliance with input, i.e. whether the agent followed the person's advice, was mentioned by approximately 3/4 of participants to lower frustration, over half the participants to increase perceived intelligence, and 1/4 of participants to increase transparency.

P9, Frustration "Frustration came from not knowing if the agent was actually taking my input and changing the way it was behaving because of it. (ie. talking to a wall)"

P22, Intelligence "I judged its intelligence on how well it mimicked what I told it to do."

P1, Transparency "It went the way I told it to quickly, and I could anticipate when to give an effective command (timing easy to ascertain)."

Essentially, an agent that does what the person wants in the way the person wants it done will create a better user experience than an agent that seems to ignore human input. This may seem somewhat obvious from a human factors perspective; however, this is not at all representative of current algorithmic design in the Interactive RL field. Personalization by endusers, i.e. having the agent perform a task in the way a human desires, can lead to the agent following policies that are technically suboptimal from an analysis of the ML metrics. For example, a path in Radiation World that avoids the radiation is slightly longer and will therefore earn a lower reward per episode than an agent that takes the shortest path right next to the radiation. In a real-life scenario, most people would be willing to take a few extra steps in order to keep a thick wall between them and a radiation leak. However, since this is not built into the reward function, an agent that solely maximizes reward will not settle on a policy that takes the slightly longer, safer path. There is a difference in this scenario between what the agent thinks is best based on its built-in reward function and what the human thinks is best based on more experience and knowledge than is incorporated into the reward function.
If people want an agent to follow instructions regardless of the underlying reward function, then one might ask: Why use Reinforcement Learning in an interactive human-agent system? Using an underlying RL algorithm enables the agent to learn when no human input is provided. It is likely that people will not provide exhaustive instructions, so the agent will need to figure out how to act to the best of its abilities in parts of the state space where no human instruction is given. For an in-depth discussion on personalization with RL, please see the paper that develops the Newtonian Action Advice algorithm [16].

This entire 'compliance with input' topic points to a larger issue within the verification of interactive RL algorithms: the field has not traditionally compared algorithms based on human factors metrics, but rather relies only on objective RL metrics.

\section{Immediacy}

An agent that immediately followed a participant's advice was mentioned by approximately half of participants as improving the perceived intelligence and transparency of the agent, and it decreased the frustration of almost $2 / 5$ of participants.

P15, Frustration "The delay between my instruction and when they performed the action."

P5, Frustration "It was very slow in responding to commands and/or ignored commands."

P9, Intelligence "A more intelligent agent seemed to respond quicker."

P19, Transparency "The ones that responded quickly seemed to use feedback most clearly."

Much research in the IML field is focused on improving agents that learn from critique. Critique algorithms use human feedback about past actions and currently have no way to impact the agent's actions in the immediate future. We know from the results of this study that an agent that does not immediately respond to the human's input will cause the user experience to suffer in numerous ways. We want to make sure that IML researchers do not 'go down the rabbit hole' of iterating over algorithms with interaction methods like critique that, by their inherent nature, may create a poor experience.

\section{Amount of instruction}

Almost $30 \%$ of participants mentioned that they thought an agent was more intelligent and transparent if the agent was able to learn from less advice given by the human teacher.

P12, Intelligence "Much less direction required to get it on the correct path."

P15, Intelligence "One agent required me to give it instruction every move which seemed less intelligent."

24, Transparency "One agent responded with less reinforcing instructions."

A key area of research through all of machine learning is generalization - making one decision apply to other similar situations in the future. This area of study is important when designing IML algorithms as it decreases the human's workload. 


\section{Randomness}

A little over $20 \%$ of the participants wrote that the agent acting randomly caused the person to be more frustrated and think of the agent as less transparent.

P8, Frustration "If the agent seemed to be guessing random directions to go if it didn't know, rather than just staying the last direction I had given it, it was frustrating."

P19, Transparency “The ones that didn't seem to try random things on their own when I wasn't talking seemed to use feedback most clearly."

P7, Transparency "When I could predict what telling it something would make it do, or if I could figure out what I'd need to tell it to get it to do what I wanted it to."

P5, Transparency "If I could predict the agents next move even if it wasn't responding to me accurately."

It is ironic that nearly all modern A.I. that can approach or surpass human performance on tasks is built on statistical theory and probabilistic models, and yet the statistical nature of agents causes people to view them as unpredictable and frustrating, which can lead to feelings of mistrust. The underlying ML agent in an interactive system must maintain its probabilistic nature to achieve reasonable levels of performance in environments with uncertainty and noise, yet the interaction between the human and agent should seem deterministic to the human in order to create a positive user experience.

Creating a deterministic interaction with the human may seem like a common sense result from a human factors perspective, yet it has much deeper consequences for the design process and is contrary to the 'common sense' of nearly all modern ML algorithm designers.

\section{Improvement through time}

Whether the agent's performance improved through time was mentioned as a contributing factor by $1 / 5$ of participants for how intelligent the agent was perceived to be. One participant thought one agent was more intelligent than others if it showed "improvement over repeated tries," while another rated intelligence based on, "if the agent began to predict where I wanted it to move."

\section{Memory}

If the agent remembered the advice, some participants mentioned that contributed to perceived intelligence and transparency.

P22, Intelligence "If one remembers what to do between two loops better than the other, I thought that it was a more intelligent agent."

P20, Transparency "The agent would use the exact advice given from prior runs to achieve the goal."

\section{Design recommendations}

When designing IML advice algorithms, we suggest the following from the results of our study:

- Minimize the time delay between when advice is given by the person and used by the agent.
- Create an interaction that is deterministic from the human's perspective rather than probabilistic.

- While the generalization of advice through the next 5 time steps rather than using advice for only one time step did not have a significant effect in terms of human factors in this case, it did enable participants to train an agent with less advice. It is possible for generalizing advice through time to decrease the amount of effort and instruction required by the human to train the agent, as well as decrease frustration by enabling people to repeat themselves less, particularly in more complex domains. However, as generalizing advice through time was not shown to significantly impact the user experience, we recommend prioritizing the creation of a minimal time delay and deterministic interaction.

\section{CONCLUSION}

Interactive Reinforcement Learning is a relatively new field, and how the nature of the interaction impacts the user experience is not widely studied. Our aim for this study is to help inform IML designers of the ramifications of their interaction design decisions. By isolating specific design decisions that impact the user's experience, IML researchers can design algorithms that create a positive user experience.

This paper focuses on the question: Do the following three factors of an agent's interaction design affect the person's experience with the agent: 1) generalization through time, 2) time delay, and 3) a probabilistic interface? If so, to what extent? The Time Delay and Probabilistic interfaces had a significant, detrimental effect on the human experience. We found the agent that generalized advice through five steps immediately in the future and the agent that had no generalization through time performed the best, both in terms of human factors and objective metrics. While both the Time Delay and Probabilistic agents created a worse experience, the Time Delay had the most negative impact on both the user experience and objective metrics.

In order to create a transparent user interaction with low frustration, high perceived intelligence, and high perceived performance, we recommend creating interfaces that have a deterministic interaction with the person and a minimal time delay between when advice is given to and used by the agent. To decrease training time, train with less advice, train with fewer steps to task completion, and create an 'instant gratification' interaction that is perceived to respond immediately to a human's instruction, it is especially important to minimize the time delay between when advice is provided and used, but it is also recommended to have a deterministic interface.

If you consider an agent to be 'liked' by a person if it scores well on human factors metrics, then the results of this study show that the human-agent team performs worse on algorithms people like the least. The agents that scored the best on the human factors metrics - the 5 steps and 1 step (no generalization) agents - also scored the best on objective machine learning metrics. 


\section{REFERENCES}

[1] Saleema Amershi, Maya Cakmak, William Bradley Knox, and Todd Kulesza. 2014. Power to the people: The role of humans in interactive machine learning. AI Magazine 35, 4 (2014), 105-120.

[2] Brenna D Argall, Brett Browning, and Manuela Veloso. 2008. Learning robot motion control with demonstration and advice-operators. In Intelligent Robots and Systems, 2008. IROS 2008. IEEE/RSJ International Conference on. IEEE, 399-404.

[3] Thomas Cederborg, Ishaan Grover, Charles L Isbell, and Andrea Lockerd Thomaz. 2015. Policy Shaping with Human Teachers.. In IJCAI. 3366-3372.

[4] Glorianna Davenport, Lars Erik Holmquist, and Maureen Thomas. 1998. Fun: A condition of creative research. IEEE MultiMedia 3 (1998), 10-15.

[5] Richard Dearden, Nir Friedman, and Stuart Russell. 1998. Bayesian Q-learning. In AAAI/IAAI. 761-768.

[6] Shane Griffith, Kaushik Subramanian, Jonathan Scholz, Charles Isbell, and Andrea L Thomaz. 2013. Policy shaping: Integrating human feedback with reinforcement learning. In Advances in Neural Information Processing Systems. 2625-2633.

[7] Sandra G Hart. 2006. NASA-task load index (NASA-TLX); 20 years later. In Proceedings of the human factors and ergonomics society annual meeting, Vol. 50. Sage Publications Sage CA: Los Angeles, CA, 904-908.

[8] David Huggins-Daines, Mohit Kumar, Arthur Chan, Alan W Black, Mosur Ravishankar, and Alexander I Rudnicky. 2006. Pocketsphinx: A free, real-time continuous speech recognition system for hand-held devices. In Acoustics, Speech and Signal Processing, 2006. ICASSP 2006 Proceedings. 2006 IEEE International Conference on, Vol. 1. IEEE, I-I.

[9] Charles Isbell, Christian R Shelton, Michael Kearns, Satinder Singh, and Peter Stone. 2001. A social reinforcement learning agent. In Proceedings of the fifth international conference on Autonomous agents. ACM, 377-384.

[10] Charles Lee Isbell, Michael Kearns, Satinder Singh, Christian R Shelton, Peter Stone, and Dave Kormann. 2006. Cobot in LambdaMOO: An adaptive social statistics agent. Autonomous Agents and Multi-Agent Systems 13, 3 (2006), 327-354.

[11] Madhura Joshi, Rakesh Khobragade, Saurabh Sarda, Umesh Deshpande, and Swati Mohan. 2012. Object-Oriented Representation and Hierarchical Reinforcement Learning in Infinite Mario. In Tools with Artificial Intelligence (ICTAI), 2012 IEEE 24th International Conference on, Vol. 1. IEEE, 1076-1081.

[12] W Bradley Knox and Peter Stone. 2010. Combining manual feedback with subsequent MDP reward signals for reinforcement learning. In Proceedings of the 9th
International Conference on Autonomous Agents and Multiagent Systems: volume 1-Volume 1. International Foundation for Autonomous Agents and Multiagent Systems, 5-12.

[13] Christin Kohrs, Nicole Angenstein, and André Brechmann. 2016. Delays in human-computer interaction and their effects on brain activity. PloS one 11, 1 (2016), e0146250.

[14] Samantha Krening and Karen M. Feigh. 2018a. Characteristics that Influence Perceived Intelligence in AI Design. In Proceedings of the Human Factors and Ergonomics Society Annual Meeting.

[15] Samantha Krening and Karen M. Feigh. 2018b. Interaction Algorithm Effect on Human Experience with Reinforcement Learning. Transactions on Human-Robot Interaction 1, 1 (2018).

[16] Samantha Krening and Karen M. Feigh. 2019. Newtonian Action Advice: Integrating Human Verbal Instruction with Reinforcement Learning. In Proceedings of the 18th International Conference on Autonomous Agents and MultiAgent Systems. International Foundation for Autonomous Agents and Multiagent Systems.

[17] Samantha Krening, Brent Harrison, Karen M Feigh, Charles Isbell, and Andrea Thomaz. 2016.

Object-Focused Advice in Reinforcement Learning. In Proceedings of the 2016 International Conference on Autonomous Agents \& Multiagent Systems. International Foundation for Autonomous Agents and Multiagent Systems, 1447-1448.

[18] Gregory Kuhlmann, Peter Stone, Raymond Mooney, and Jude Shavlik. 2004. Guiding a reinforcement learner with natural language advice: Initial results in RoboCup soccer. In The AAAI-2004 workshop on supervisory control of learning and adaptive systems.

[19] James MacGlashan, Monica Babes-Vroman, Marie desJardins, Michael Littman, Smaranda Muresan, Shawn Squire, Stefanie Tellex, Dilip Arumugam, and Lei Yang. 2015. Grounding English Commands to Reward Functions. In Proceedings of Robotics: Science and Systems. Rome, Italy.

[20] Richard Maclin, Jude Shavlik, Lisa Torrey, Trevor Walker, and Edward Wild. 2005. Giving advice about preferred actions to reinforcement learners via knowledge-based kernel regression. In Proceedings of the National Conference on Artificial intelligence, Vol. 20. Menlo Park, CA; Cambridge, MA; London; AAAI Press; MIT Press; 1999, 819.

[21] Cetin Meriçli, Steven D Klee, Jack Paparian, and Manuela Veloso. 2014. An interactive approach for situated task specification through verbal instructions. In Proceedings of the 2014 international conference on Autonomous agents and multi-agent systems. International Foundation for Autonomous Agents and Multiagent Systems, 1069-1076. 
[22] Neema Moraveji and Charlton Soesanto. 2012. Towards stress-less user interfaces: 10 design heuristics based on the psychophysiology of stress. In CHI'12 extended abstracts on Human factors in computing systems. ACM, 1643-1648.

[23] Raja Parasuraman and Victor Riley. 1997. Humans and automation: Use, misuse, disuse, abuse. Human factors 39, 2 (1997), 230-253.

[24] Himanshu Sahni, Brent Harrison, Kaushik Subramanian, Thomas Cederborg, Charles Isbell, and Andrea Thomaz. 2016. Policy shaping in domains with multiple optimal policies. In Proceedings of the 2016 International Conference on AAMAS. International Foundation for AAMAS, 1455-1456.

[25] Ben Shneiderman. 1984. Response time and display rate in human performance with computers. $A C M$ Computing Surveys (CSUR) 16, 3 (1984), 265-285.

[26] Manimaran Sivasamy Sivamurugan and Balaraman Ravindran. 2012. Instructing a Reinforcement Learner. In FLAIRS Conference.

[27] Burrhus Frederic Skinner. 1938. The behavior of organisms: An experimental analysis. (1938).
[28] Burrhus F Skinner. 1981. Selection by consequences. Science 213, 4507 (1981), 501-504.

[29] Michael Thomas Stokes. 1990. Time in human-computer interaction: performance as a function of delay type, delay duration, and task difficulty. Ph.D. Dissertation. Texas Tech University.

[30] Kaushik Subramanian, Charles L Isbell Jr, and Andrea L Thomaz. 2016. Exploration from demonstration for interactive reinforcement learning. In Proceedings of the 2016 International Conference on AAMAS. International Foundation for AAMAS, 447-456.

[31] Richard S Sutton and Andrew G Barto. 1998. Reinforcement learning: An introduction. Vol. 1. MIT press Cambridge.

[32] Stefanie Tellex, Thomas Kollar, Steven Dickerson, Matthew R Walter, Ashis Gopal Banerjee, Seth J Teller, and Nicholas Roy. 2011. Understanding Natural Language Commands for Robotic Navigation and Mobile Manipulation.. In AAAI, Vol. 1. 2.

[33] Andrea L Thomaz and Cynthia Breazeal. 2008. Teachable robots: Understanding human teaching behavior to build more effective robot learners. Artificial Intelligence 172, 6-7 (2008), 716-737. 